\title{
Carrier Density and Schottky Barrier on the Performance of DC Nanogenerator
}

\author{
Jin Liu, ${ }^{\dagger}$ Peng Fei, ${ }^{\dagger, \ddagger}$ Jinhui Song, ${ }^{\dagger}$ Xudong Wang, ${ }^{\dagger}$ Changshi Lao, ${ }^{\dagger}$ \\ Rao Tummala, ${ }^{\dagger}$ and Zhong Lin Wang ${ }^{\star, \dagger}$ \\ School of Materials Science and Engineering, Georgia Institute of Technology, \\ Atlanta, Georgia 30332-0245, and Department of Advanced Materials and \\ Nanotechnology, College of Engineering, Peking University, 100084 Beijing, China
}

Received November 1, 2007; Revised Manuscript Received December 5, 2007

\begin{abstract}
By assembling a ZnO nanowire (NW) array based nanogenerator (NG) that is transparent to UV light, we have investigated the performance of the NG by tuning its carrier density and the characteristics of the Schottky barrier at the interface between the metal electrode and the NW. The formation of a Schottky diode at the interface is a must for the effective operation of the NG. UV light not only increases the carrier density in $\mathrm{ZnO}$ but also reduces the barrier height. A reduced barrier height greatly weakens the function of the barrier for preserving the piezoelectric potential in the NW for an extended period of time, resulting in little output current. An increased carrier density speeds up the rate at which the piezoelectric charges are screened/neutralized, but a very low carrier density prevents the flow of current through the NWs. Therefore, there is an optimum conductance of the NW for maximizing the output of the NG. Our study provides solid evidence to further prove the mechanism proposed for the piezoelectric NG and piezotronics. The output current density of the NG has been improved to 8.3 $\mu \mathrm{A} / \mathrm{cm}^{2}$.
\end{abstract}

Developing nanomaterial-enabled technologies for energy harvesting has attracted a lot of interest recently., ${ }^{1,2}$ Using aligned $\mathrm{ZnO}$ nanowire (NW) arrays, we have recently demonstrated a nanogenerator (NG) for converting mechanical energy into electricity. ${ }^{3,4}$ The mechanism of the NG relies on the coupled semiconducting and piezoelectric properties and is composed of two processes. ${ }^{5,6}$ When a clean $\mathrm{ZnO}$ NW or nanobelt is bent by an atomic force microscope (AFM) tip, an asymmetric strain is produced across the width of the NW. As a result of piezoelectricity, the stretched side of the NW has a positive potential and its compressed side has a negative potential. The contact between a Pt coated tip with $\mathrm{ZnO}$ is a Schottky diode. When the tip contacts the NW and bends it, the contact between the tip and the stretched side is a reversely biased Schottky diode. In such a case, a piezoelectric potential is created in the NW, but there is no charge flowing across the Schottky diode although there is a piezoelectric potential in the NW side, resulting in a charge creation and accumulation. This is the first process. When the tip scans in contact mode and reaches the compressed side of the NW, a forward biased Schottky diode is formed at the interface; thus, the external electrons can flow across the interface under the driving of the piezoelectric potential, resulting in an external current

* To whom correspondence should be addressed. E-mail: zhong.wang@ mse.gatech.edu.

Georgia Institute of Technology.

$\doteqdot$ Peking University. detected by the measurement meter. This is the current output process.

The mechanism of the NG is based on two important physical quantities. One is the height of the Schottky barrier, which needs to be high enough to hold the charges from leaking. Second, the conductivity and carrier density of the $\mathrm{ZnO} \mathrm{NW}$ are adequately low in the first step to preserve the piezoelectric potential distribution in the NW from being "neutralized" by the freely flowing charge carriers, which are electrons for n-type $\mathrm{ZnO}$, but they need to be high enough to transport the current under the driving of the piezoelectric potential in the charge releasing process. In this paper, we have designed a series of experiments to tune the height of the Schottky barrier and the carrier density by UV irradiation to study their effects on the performance of the NG. We experimentally show that a Schottky diode between the top metal electrode and the NW arrays is a must for the NG, whereas the carrier density has an optimum value at which the output of the NG is expected to be maximized. A testing criterion is demonstrated for determining the effectiveness of a NG for generating current.

Experimental Design. The aligned NW arrays to be used for the experiments were grown epitaxially on an optically transparent AlN/sapphire substrate using a method reported elsewhere. ${ }^{7}$ The bottom of the substrate was polished for enhancing optical transparency. At the roots of the NWs, a thin layer of $\mathrm{ZnO}$ film was also grown simultaneously, which served as a common electrode for electrical measurements. 

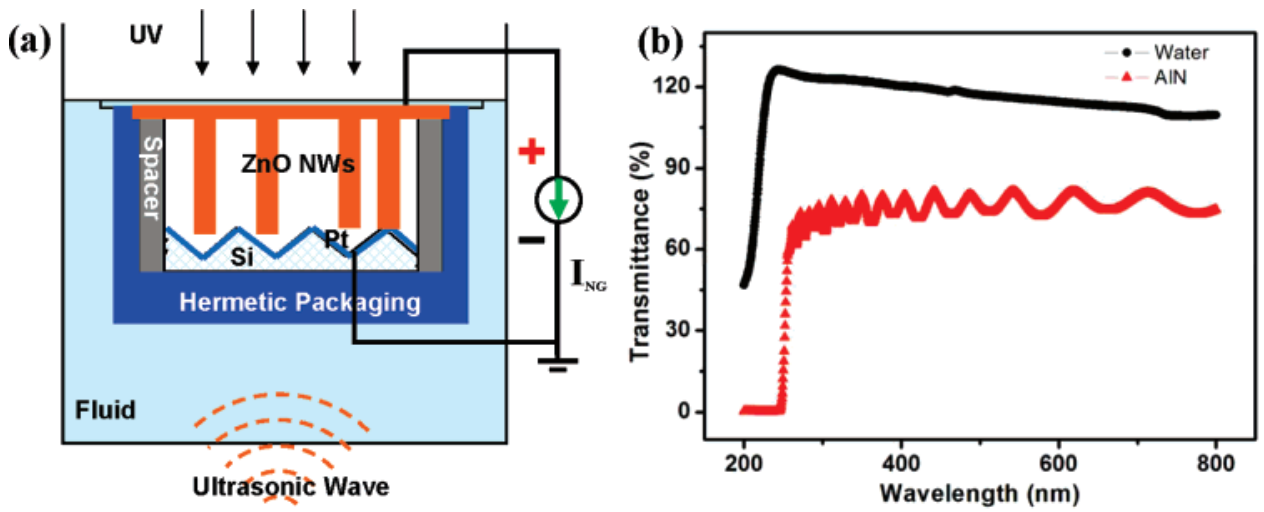

Figure 1. (a) Experimental set up for measuring the UV response of an assembled NG in liquid. The NG is placed up side down to ensure the UV illumination from the substrate side to reach the ZnO NWs. (b) UV transmittance spectra through AlN/sapphire substrate and water to ensure the penetration of the UV into the core of the NG.
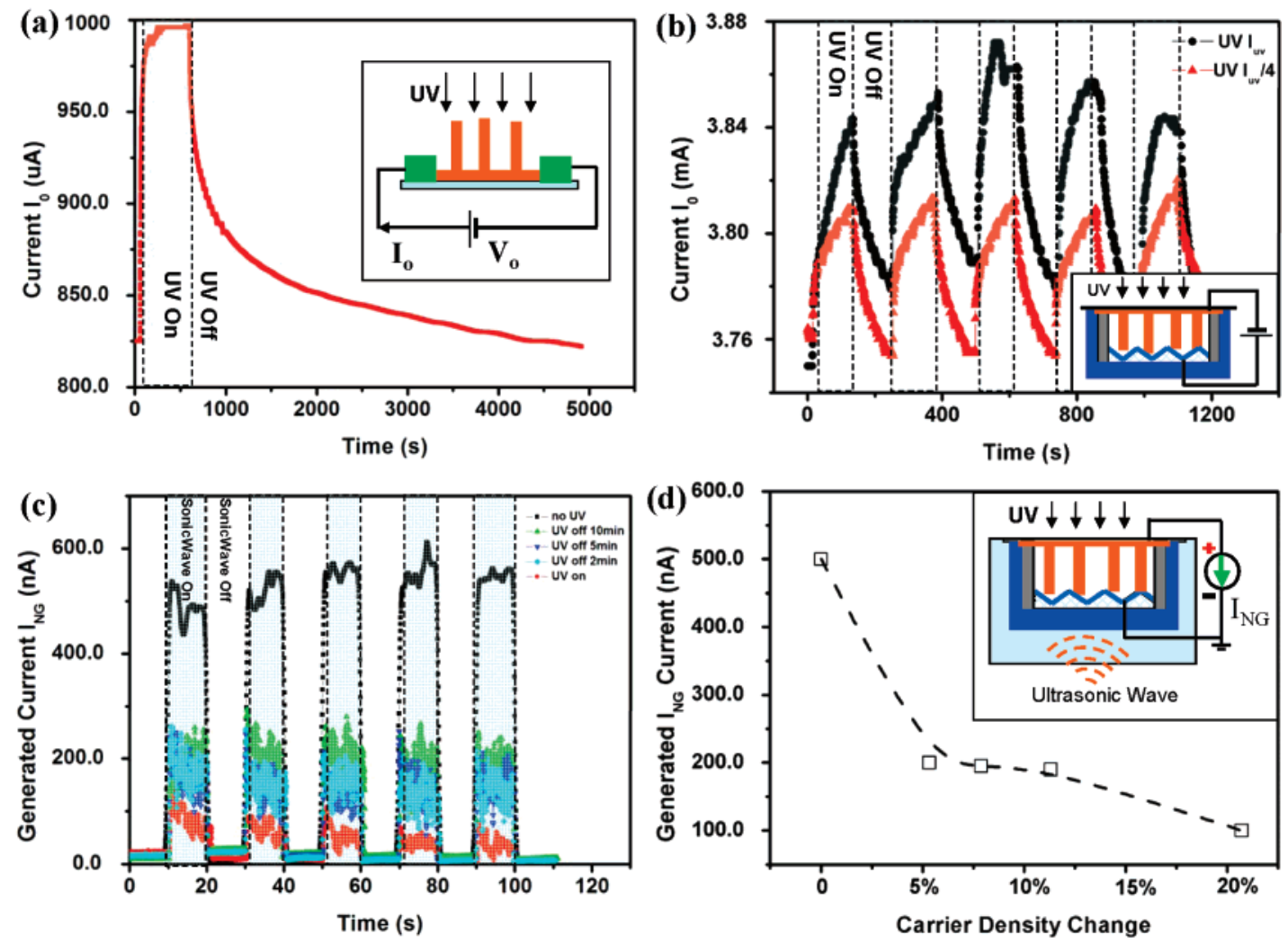

Figure 2. Tuning the transport property of $\mathrm{ZnO}$ and an assembled NG by UV excitation. (a) Response of the current transported through a thin ZnO film with NWs on top when subjecting to UV illumination, showing a long delay in the recovering process. The externally applied voltage $V_{0}=2 \mathrm{~V}$. The inset is the measurement set up. (b) Current transported through an assembled NG under an externally applied voltage $V_{0}=2 \mathrm{~V}$ when the UV light was tuned on and off. The dark and red curves represent the response curve at full UV intensity $I_{\mathrm{UV}}$ and $I_{\mathrm{UV}} / 4$, respectively. The inset is the measurement set up. Response of the NG output subjecting to UV excitation. (c) Current generated by the NG when the ultrasonic wave was turned on and off under different illumination conditions of UV light (see text). A dramatic fact is that the UV illumination reduces the output current to $10-20 \%$. The size of the NG is $6 \mathrm{~mm}^{2}$. (d) Plot of the NG generated current as a function of the increased percentage of carried density in the NWs. The inset is the measurement set up.

Our fabrication of DC NG follows the methodology developed in ref 4. A Pt coated Si zigzag electrode was aligned on top of the ZnO NWs. A flexible spacer was placed between the top electrode and the AlN/sapphire substrate to ensure a proper space required for the NG. The device was then packaged with a water-proof polymer. The entire NG was mounted on a UV-transparent quartz slide (SPI 01016$\mathrm{AB}$ quartz slide) before testing. The packaged NG was placed upside down in a well isolated water bath, with the quartz slide and AlN/substrate facing up so that the UV light was able to penetrate the slide and reach the core of the NG, as shown in Figure 1a. The UV light source (100 W long wave mercury spot lamp) was placed $5 \mathrm{~cm}$ above the water surface. A $41 \mathrm{kHz}$ ultrasonic wave generator was used as the source to excite the NG from the bottom of the water bath.

The transmission spectrum from 200 to $800 \mathrm{~nm}$ was measured to ensure that the UV reached the $\mathrm{ZnO} \mathrm{NWs}$, as shown in Figure 1b. Then the UV transmittance of the AlN/ 


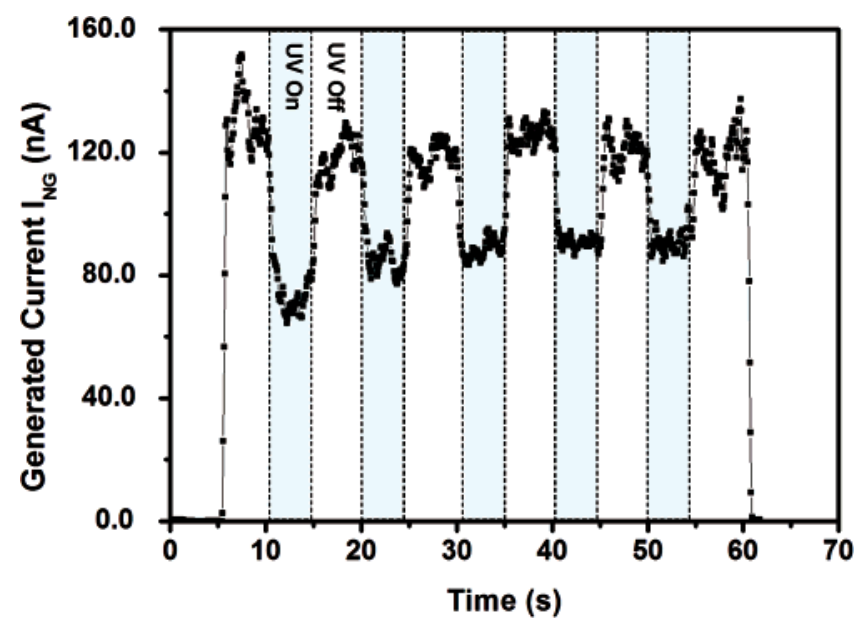

Figure 3. Output of a NG as excited by ultrasonic wave when the UV light was turned on and off. The UV light can reduce the output current $30-45 \%$, indicating increasing of carrier density is not beneficial for improving the output power.

sapphire substrate was measured by stacking the substrate on a UV transparent quartz slide, as shown by the red curve in Figure 1b, where the oscillation in the measured data is due to the interference of the light reflected from the substrate and the slide. Finally, the UV transmittance was measured for two parallel plates filled with water between them. Due to the reduced total reflection in the water and plate interface, the measured transmittance is even better than that without water (>100\%; dark line). Our measurement data clearly show that the near UV light can effectively penetrated through the substrate and water to reach the ZnO NWs.

Carrier Density on the Output of NG. To evaluate the UV response of $\mathrm{ZnO} \mathrm{NWs}$ as well as an assembled NG, two experiments have been carried out. Figure 2 a shows the $\mathrm{UV}$ response of the vertically aligned $\mathrm{ZnO} \mathrm{NWs}$ and the underlying $\mathrm{ZnO}$ thin film by connecting two electrodes at the two ends of the film, as shown in the inset. By applying a constant voltage $V_{0}$, the transport current was monitored when the UV was turned on or off. The conductance of the film responded rapidly to UV and reached saturation after $\sim 300 \mathrm{~s}$. However, the current decayed very slowly after the UV was turned off, possibly due to the following reason. The UV light can effectively create electron-hole pairs in $\mathrm{ZnO}$ and greatly increase the carrier density. After turning off the UV, the electrons and holes would recombine and thus decrease the conductivity. However, some carriers may be trapped in the surface/vacancy states in $\mathrm{ZnO}$, which greatly delayed the electron-hole recombination rate, possibly resulting in a long decay in conductivity.

The long lasting tail in the recovery of conductivity in Figure 2a may possibly indicate that $\mathrm{ZnO}$ can hold the charges for an extended period of time before they are completely and entire neutralized/screened by external electrons. One may suggest that the piezoelectric charges and potential created in a NW may hold for an equivalent length of time before being neutralized/screened by external electrons, so that it can effectively gate the charge carriers as proposed for nanopiezotronics, ${ }^{8}$ giving sufficient time for experimental observation of the effect of the piezoelectric
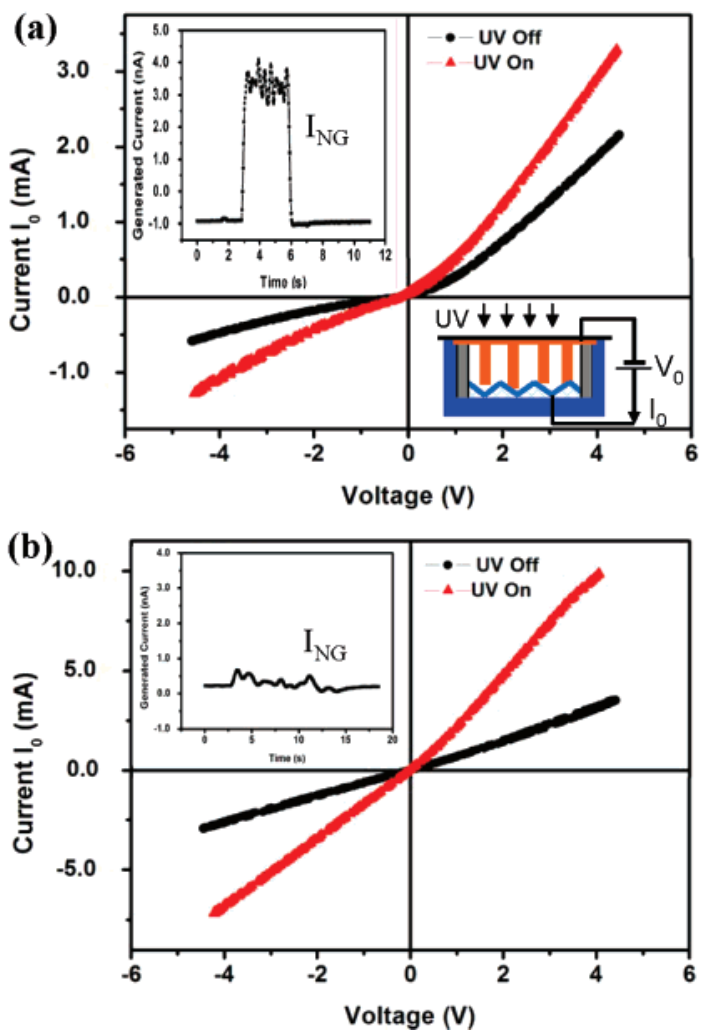

Figure 4. $I-V$ characteristic of an assembled NG for identifying its performance for producing current. (a) For an NG that actively generating current, as shown in the inset, its I-V curve when the ultrasonic wave was off clearly shows a Schottky diode behavior. UV light not only increased the carrier density (or conductivity) but also might reduce the barrier height. (b) For a "defective" NG that did not produce current, the I-V curve shows a clearly Ohmic behavior. The UV light clearly increased the conductivity.

potential across a NW. Such length of time may be even longer than tens to hundreds of seconds.

The second experiment was to characterize the UV response of an assembled NG, as shown in the inset in Figure $2 \mathrm{~b}$. By reducing the relative UV intensity from $I_{\mathrm{UV}}$ to $\sim I_{\mathrm{UV}} / 4$, the conductance of the NG changed for $\sim 2 \%$. The conductivity also sharply responded to the on and off of the UV, clearly showing that the UV can effectively increase the carrier density in the NG.

After observing the increased conductivity in NG under UV irradiation, we investigated the performance of the NG for generating electricity. Figure $2 \mathrm{c}$ is the generated current $\left(I_{\mathrm{NG}}\right)$ of a NG when subjecting to ultrasonic wave excitation by changing the UV irradiating condition. When the UV light was off for an extended period of time, the generated current was $\sim 500 \mathrm{nA}$ for a NG with $6 \mathrm{~mm}^{2}$ in size, corresponding to an output current density of $8.3 \mu \mathrm{A} / \mathrm{cm}^{2}$. This value is about 20 times higher than that reported previously. ${ }^{6}$ The key to obtaining a high output current density is to have NWs that are uniform in size and with patterned distribution on substrate that matches the design of the electrode. As soon as the UV light was turned on, the generated current dropped to as low as $30-80 \mathrm{nA}$. At the same time, we monitored the generated current $I_{\mathrm{NG}}$ after the UV was turned off for 2, 5, and $10 \mathrm{~min}$, and the generated current increased to $\sim 180$, 190, and $205 \mathrm{nA}$, respectively. Such a gradual increase in 

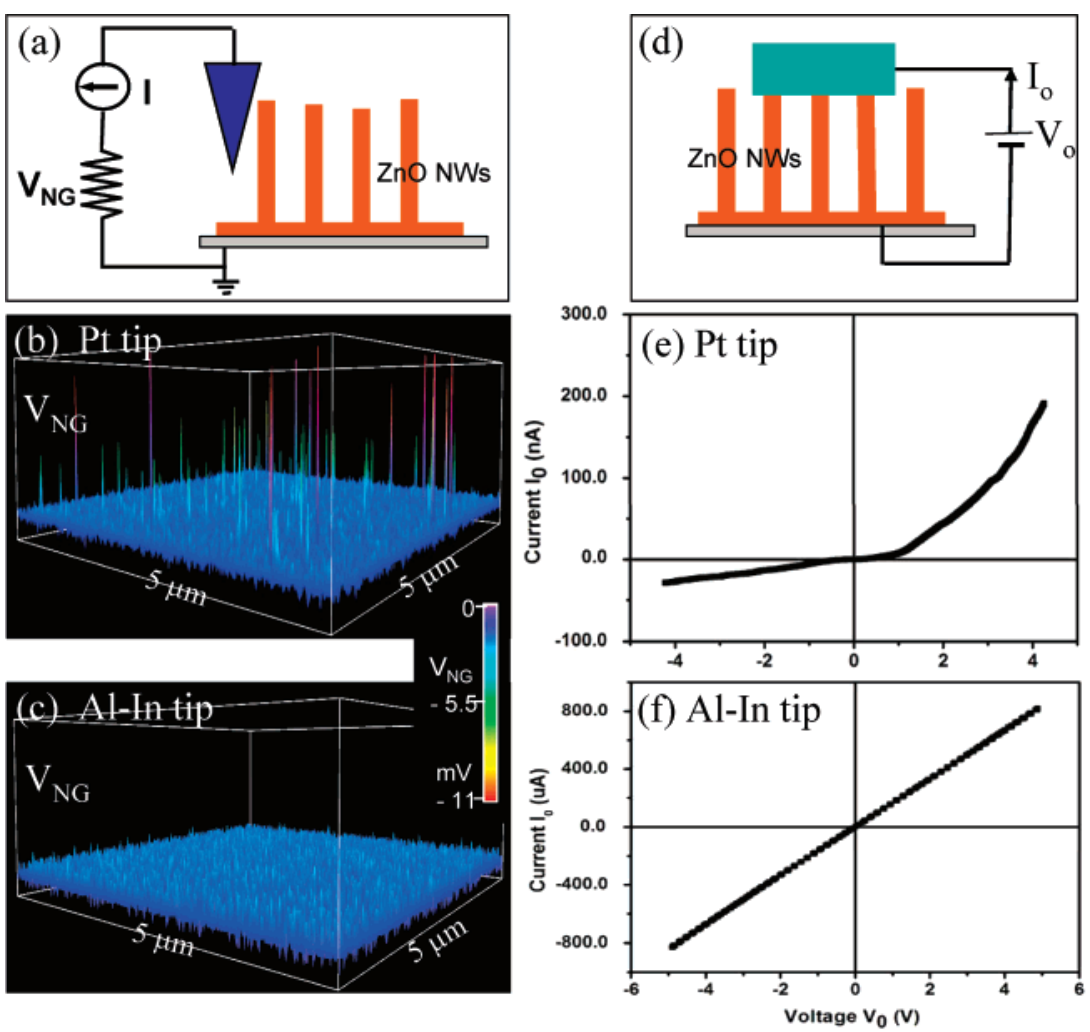

Figure 5. (a) AFM based measurement set up for correlating the relationship between the metal-ZnO contact and the NG output. (b) Output potential generated by ZnO NW array when scanned by a Pt coated Si tip. (c) No output potential is generated by ZnO NW array when scanned by an $\mathrm{Al}-\mathrm{In}$ coated Si tip. (d) Experimental set up for characterizing the I-V transport property of metal-ZnO NW contact. (e) $\mathrm{I}-\mathrm{V}$ curve of a $\mathrm{Pt}-\mathrm{ZnO} \mathrm{NW}$ contact, showing Schottky diode effect. (f) I-V curve of an alloyed Al/In $-\mathrm{ZnO} \mathrm{NW}$ contact, showing Ohmic behavior.

the generated current corresponds to the slow decay in conductivity as shown in Figure 2b. This set of experiments apparently shows that an increase in conductivity, e.g., carrier density, reduces the output current of the NG. By extracting the relative change in carrier density from Figure $2 \mathrm{a}$ and the correspondingly measured NG output $I_{\mathrm{NG}}$ from Figure 2c, Figure $2 \mathrm{~d}$ illustrates the monotone decrease relationship between the increased percentage in carrier density and the output current $I_{\mathrm{NG}}$. This result indicates that the carrier density in $\mathrm{ZnO}$ is one of the key characteristics that dictate the output power of a NG. A too high carrier density would screen the piezoelectric charges, resulting in a lower or even vanishing output current. However, a too low carrier density would increase the inner resistance and greatly decrease the output current. Therefore, there should be an optimum choice of NW carrier density or conductivity to maximize the NG output.

In a separate experiment, the output of a NG was monitored when the ultrasonic wave was continuously on but with the UV being turned on and off, as shown in Figure 3 . The data reproducibly show that UV irradiation reduced the output current by $30-45 \%$.

Schottky Barrier on the Performance of the NG. The Schottky contact between the metal contact and $\mathrm{ZnO}$ NW is another key factor to the current generation process. Investigating how it affects the performance of NG will provide effective guidance in designing and fabricating high output NGs. We characterized the $\mathrm{I}-\mathrm{V}$ characteristic of a set of
NGs to illustrate the effects of Schottky barrier for current generation. Figure $4 a$ shows the $\mathrm{I}-\mathrm{V}$ characteristics of a NG that did generate output current (top inset of Figure 4a). The experimental setup for the measurement is show in the inset at the lower right corner in Figure 4a. The NG exhibits a Schottky-like I-V characteristic, which means that the NG has different responses for forward and reverse biases. When being illuminated by UV, the NG showed an increased conductance, and its Schottky-like rectifying effect was largely reduced due to the increased conductance. Alternatively, for a "defective" NG that did not produce current in responding to ultrasonic wave, its $\mathrm{I}-\mathrm{V}$ characteristic was clearly Ohmic even prior to UV illumination (Figure 4b). By examining over $10 \mathrm{NGs}$, their $\mathrm{I}-\mathrm{V}$ characteristics reliably indicated if the NGs were effective for producing current. This study establishes a criterion for identifying the working NG from the defective ones.

To examine the role played by the Schottky barrier in the NG, we used the AFM based manipulation and measurement system as used in our first study for demonstrating the piezoelectric $\mathrm{NG}^{3}$ (see Figure 5a). When a $100 \mathrm{~nm}$ thick $\mathrm{Pt}$ coated Si tip was used for scanning the NWs in contact mode, voltage peaks were observed (Figure 5b), and the output voltages were in the order of $\sim-11 \mathrm{mV}$ (the negative sign means that the current flowed from the grounded end through the external load). By changing the tip to an $\mathrm{Al}-\mathrm{In}(30$ $\mathrm{nm} / 30 \mathrm{~nm}$ ) alloy coated Si tip, the $\mathrm{ZnO}$ NWs showed no piezoelectric output (Figure 5c). In order to understand the 


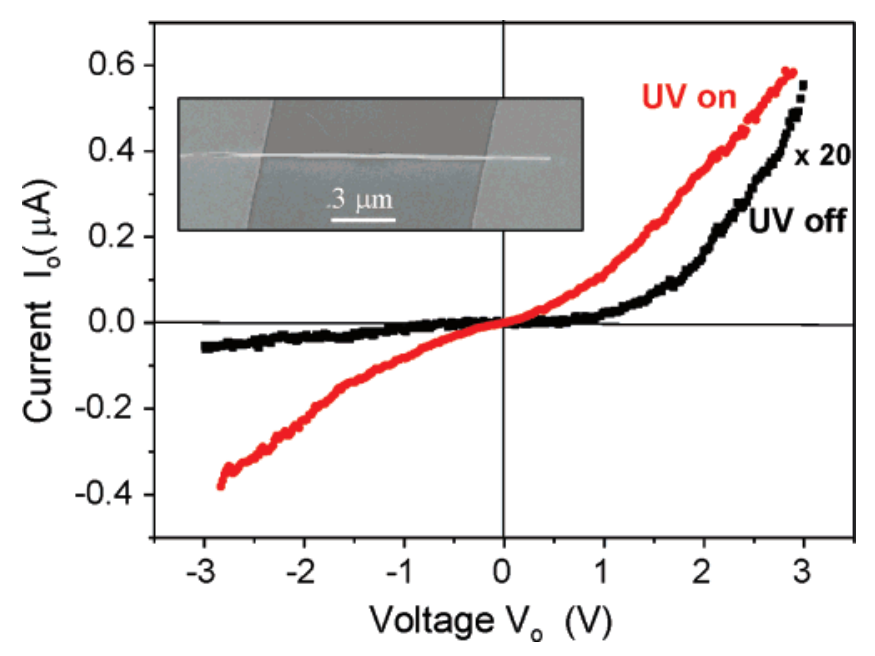

Figure 6. Two-electrode-based device fabricated using a $\mathrm{ZnO} N W$ for measuring its transport property when subjecting to UV illumination. An SEM image of the device is inset. The asymmetric contact at the two ends results in a Schottky diode. The UV not only increased the carrier density but also reduced the barrier height at the interface.

two distinct performances of the two types of scanning tips, we have measured their corresponding $\mathrm{I}-\mathrm{V}$ characteristics with the $\mathrm{ZnO}$ NWs. To ensure the stability of the contact, we used a large electrode that was in contact with a group of NWs, as shown in Figure 5d. The Pt- $\mathrm{ZnO}$ contact clearly presented a Schottky diode (Figure 5e), whereas the Al$\mathrm{In}-\mathrm{ZnO}$ was an Ohmic contact. In reference to the piezoelectric output presented in Figure 5b,c, we conclude that a Schottky contact between the metal electrode and $\mathrm{ZnO}$ is a must for a working NG.

UV Illumination on the Performance of the Schottky Barrier. As illustrated in the last section, the presence of a Schottky barrier between the metal electrode and the $\mathrm{ZnO}$ NWs is a key for determining the performance of the NG. We now examine the influence of UV light on the performance of a Schottky diode. For this purpose, we used a pair of $\mathrm{Au}$ electrodes interconnected by a $\mathrm{ZnO} \mathrm{NW}$, and a Schottky diode based device was fabricated (inset in Figure 6). The Schottky diode characteristic of the NW (dark curve) may be produced by the asymmetric contacts of the $\mathrm{ZnO}$ NW with the two electrodes. ${ }^{9}$ When being illuminated by $\mathrm{UV}$, the conductance of the device was improved over 20 times (red curve in Figure 6). More importantly, the threshold voltage of the diode has been greatly reduced from close to $1 \mathrm{~V}$ (without UV) to almost 0 (with UV; Figure 6), and the I-V curve shows an Ohmic-like behavior. ${ }^{10}$ Therefore, a weakening or disappearing rectifying effect of the diode is likely to be the reason why the performance of the NG as shown in Figure 2c,d deteriorated with the increase of UV generated carrier density.

In summary, by assembling aligned $\mathrm{ZnO} \mathrm{NW}$ array based NGs that were transparent to UV light, we investigated the performance of the NG by tuning its carrier density and the transport property of the Schottky barrier between the metal electrode and the NWs. The presence of a Schottky diode between the top zigzag metal electrode and the $\mathrm{ZnO} \mathrm{NWs}$ is mandatory for the effective operation of the NG. UV light not only increases the carrier density in $\mathrm{ZnO}$ but also reduces the barrier height between the metal electrode and $\mathrm{ZnO}$. A reduced Schottky barrier height greatly weakens the function of the barrier for preserving the piezoelectric potential in the NW. A replacement of a Schottky contact by an Ohmic contact overrides the role played by the barrier for accumulating the piezoelectric charges. If there is no charge accumulation in the first process, no charge would be released in a later stage. Our study shows that identifying the existence of the Schottky diode transport property of a NG is an effective means for identifying if the NG is good or defective.

An increased carrier density speeds up the rate at which the piezoelectric charges are screened/neutralized. However, a very low carrier density may improve the local piezoelectric potential and prevents the flow of current through the NWs, as well as increases the inner resistance of the NWs and possibly the contact resistance at the interface. Therefore, there should be an optimum choice of NW conductance for maximizing the output of the NG. NWs with excellent conductivity may greatly reduce the life time of the piezoelectric charges, resulting in very small or no output current. A comprehensive computer simulation that takes into consideration the dynamics of charge transport is necessary for predicting the value of the optimum conductance.

In conclusion, our study presented in this paper provides solid evidence to fully support and prove the mechanism proposed for the piezoelectric NG and piezotronics.

Acknowledgment. Thanks to Yifan Gao, Chen Xu, Cheng Li, and Yuzi Liu for technical assistance. This work was supported by DOE BES (DE-FG02-07ER46394) and NSF (DMS 0706436).

\section{References}

(1) Tian, B.; Zheng, X.; Kempa, T. J.; Fang, Y.; Yu, N.; Yu, Y.; Huang, J. L.; Lieber, C. M. Nature 2007, 449, 885.

(2) Shi, L.; Li, D.; Yu, C.; Jang, W.; Kim, D.; Yao, Z.; Kim, P.; Majumdar, A. J. Heat Transfer 2003, 125, 881.

(3) Wang, Z. L.; Song, J. H. Science 2006, 312, 242.

(4) Wang, X. D.; Song, J. H.; Liu, J.; Wang, Z. L. Science 2007, 316, 102.

(5) Song, J. H.; Zhou, J.; Wang, Z. L. Nano Lett. 2006, 6, 1656

(6) Wang, X. D.; Liu, J.; Song, J. H.; Wang, Z. L. Nano Lett. 2007, 7, 2475.

(7) Wang, X. D.; Song, J. H.; Li, P.; Ryou, J. H.; Dupuis, R. D.; Summers, C. J.; Wang, Z. L. J. Am. Chem. Soc. 2005, 127, 7920

(8) Wang, Z. L. Adv. Mater. 2007, 19, 889.

(9) Lao, C. S.; Liu, J.; Gao, P. X.; Zhang, L. Y.; Davidovic, D.; Tummala, R.; Wang, Z. L. Nano Lett. 2006, 6, 263.

(10) Newton, M. C.; Firth, S.; Wauburton, P. A. Appl. Phys. Lett. 2006, 89, 072104.

NL0728470 